\title{
DIFUSIÓN DEL CAMBIO TECNOLÓGICO Y SU IMPACTO SOBRE EL NIVEL DE EMPLEO EN SECTORES POTENCIAL- MENTE INNOVADORES DE LA ECONOMÍA ANDALUZA (1975-1980). EVALUACIÓN Y ANÁLISIS A PARTIR DE LAS TABLAS INPUT-OUTPUT. *
}

\author{
Antonio GARCÍA SÁNCHEZ ** \\ José Luis MARTÍN NAVARRO ** \\ Luis PALMA MARTOS **
}

\section{INTRODUCCIÓN.}

El avance tecnológico constituye una de las fuentes primordiales del desarrollo de todo sistema economico. En regiones en las que la innovación tecnológica autóctona es muy limitada, el avance tecnológico y sus efectos dinamizadores sobre el tejido productivo y el empleo derivan básicamente de la adaptación y difusión de progresos tecnológicos generados en otras áreas. Creemos que este es el caso de Andalucía y por ello planteamos un estudio que nos permita aproximarnos a la medición de dicho fenómeno. De ahí el objetivo del trabajo que hemos planteado: realizar una estimacion del grado de difusi6n del cambio tecnologico en Andalucía, con especial atención a su impacto en el nivel de empleo en sectores potencialmente innovadores, a partir de las Tablas Input-Output regionales disponibles actualmente, editadas para los años 1975 y $1980^{1}$.

*. Queremos agradecer a Blas Díaz León y a Antonio Santisteban Blanco la colaboración prestada durante el desarrollo del presente trabajo.

**. Departamento de Teoría Económica y Economía Política de la Universidad de Sevilla.

1. Durante la redacción del presente trabajo ha aparecido, editada por el Instituto de Estadística de Andalucía, en edición provisional, la Tabla Input-Output de la Economía Andaluza correspondiente a 1990. En la actualidad se trabaja en una extensión del análisis incorporando los datos contenidos en la citada tabla. 
Tal como señala Stonemam (1983), existen varios modelos y metodos para abordar la cuestión que hemos planteado. Optamos por la utilización del análisịs Input-Output por constituir una de las herramientas cuyo uso está más extendido en los estudios empíricos macroeconómicos, debido a la posibilidad de integrar todas las interrelaciones y contar con una importante desagregación sectorial de la economía ${ }^{2}$.

La solución al debate sobre la incidencia del cambio tecnológico en el nivel de empleo es compleja. El efecto que la implantación de nuevas tecnologías tiene sobre el empleo, aporta un factor de preocupación social al proceso de modernización económica. Que el largo plazo sea el período a considerar para recoger los frutos de las innovaciones tecnicas en cuanto a creación de empleo, es la hipótesis esgrimida al aceptar la necesidad de los cambios técnicos.

En el presente artículo incidimos en el análisis de esta problemática, centrándonos en una serie de sectores que pueden considerarse potencialmente innovadores, utilizando los datos recogidos en las Tablas Input-Output de la economía andaluza para los años de 1975 y 1980.

Diversos trabajos han abordado el tema de la difusión tecnológica y su impacto sobre el nivel de empleo mediante el uso de las tecnicas input-output. A título de ejemplo puede verse la revisión realizada por Sato, R. y Ramachandran, R. (1980), el análisis de McNicoll, I. H. y Bairdy, R. G. (1980), y el trabajo de Afrasiabi, A. y Casler, S. D. (1991).

Para el caso español ha sido numerosos los trabajos realizados a nivel nacional y regional. Sin ánimo de ser exhaustivos podemos señalar: Fanjul et al. (1975), Segura y Restoy (1986), Saez et al. (1991a) y (1991b), Sáez (1992) e IFA (1991) referido exclusivamente al sector industrial en Andalucía.

\section{METODOLOGÍA DEL MODELO GENERAL}

Para la economía andaluza disponemos en la actualidad de una serie de dos tablas correspondientes a los años 1975 y 1980. Estos documentos presentan el inconveniente de la falta de homogeneidad en el establecimiento de Ios sectores a considerar, 30 en la tabla de 1975 y 64 en la de 1980. Por ello hemos tenido que realizar la agregación de algunos sectores de la tabla de 1980, de acuerdo con los criterios establecidos en esta última tabla (pág. 30, Anexo 2.3).

2. Puede verse una justificación del modelo Input-Output, así como una breve formulación del mismo en RAMÍREZ SOBRINO, Jesús N. (1993), pág. 127-138. 
Partimos del modelo desarrollado por Fanjul èt al. (1975). Se trata de analizar los cambios en el tiempo de los flujos intersectoriales agregados de los inputs intermedios. Estos flujos vienen medidos por $\mathrm{q}_{\mathrm{t}}$ de tal manera que

$$
\mathrm{q}_{\mathrm{t}}=\mathrm{y}_{\mathrm{t}}-\mathrm{z}_{\mathrm{t}}=\left(\mathrm{I}-\mathrm{A}_{\mathrm{t}}\right)^{-1} \cdot \mathrm{z}_{\mathrm{t}}-\mathrm{z}_{\mathrm{t}}
$$

donde $y_{t}$ es el output total, $z_{t}$ es la demanda final total y $A_{t}$ es la matriz de coeficientes interindustriales de Leontief.

Las variaciones entre $q_{t}$ y $q_{t+1}$ se pueden deber fundamentalmente a tres factores: cambios en la estructura de la demanda final, en los niveles de actividad (ambos medidos por $\mathrm{z}_{\mathrm{f}}$ ) y variaciones en la estructura técnica del sistema productivo, medidos por $\mathrm{A}_{\mathrm{t}}$. Estas últimas variaciones pueden aislarse manteniendo el vector de demanda final $\left(\mathrm{z}^{*}\right)$ constante, con lo que la ecuación (1) queda convertida en

$$
\mathrm{q}_{\mathrm{t}}{ }^{*}=\left(\mathrm{I}-\mathrm{A}_{\mathrm{t}}\right)^{-1} \cdot \mathrm{z}^{*}-\mathrm{z}^{*}
$$

En nuestra aplicación se ha optado por mantener fijo el vector de demanda de 1980.

Se han considerado las importaciones como sustitutivas de los inputs regionales, es decir, independientes del nivel de actividad regional, incorporándolas a la matriz de coeficientes técnicos. La ecuación (2) queda así transformada en

$$
\mathrm{q}_{\mathrm{t}}^{*}=\left(\mathrm{I}-\mathrm{A}_{\mathrm{t}}\right)^{-1} \cdot\left(\mathrm{z}^{*}-\mathrm{m}^{*}\right)-\mathrm{z}^{*}+\mathrm{m}^{*}
$$

$\mathrm{y} \mathrm{m}^{*}$ representa el vector de importaciones que se ha mantenido fijo en el valor correspondiente a 1980. A partir de (3) se procede a transformar en matriz diagonal el vector de demanda final $\mathrm{z}^{*}$ y a sustituir el vector de importaciones por una matriz al objeto de incorporar el flujo de inputs procedente de las importaciones y su difusion entre los diversos sectores. La expresión resultante es:

$$
Q^{*}=\left(I-A_{t}\right)^{-1} \cdot\left(Z^{*}-M^{*}\right)-Z^{*}+M^{*}
$$

donde $\mathrm{Q}^{*}$ es la matriz de necesidades de bienes intermedios de la economía andaluza, $\mathrm{Z}^{*}$ es la matriz diagonalizada correspondiente al vector $\mathrm{z}^{*}, \mathrm{y}^{*}$ es la matriz de importaciones.

Para llevar a cabo el análisis del efecto sobre el nivel de empleo, es necesario conocer el contenido tecnológico de cada uno de los sectores, definido como la proporción que el total de los inputs intermedios $\left(\mathrm{X}_{\mathrm{ij}}\right)$ procedentes de los doce 
sectores seleccionados como potencialmente innovadores, representa sobre el total de los inputs intermedios del sector.

Posteriormente se estudian las variaciones en los requerimientos de empleo de la economía regional (análisis por columnas), medidos a partir de:

$$
\mathrm{R}(1980)-\mathrm{R}(1975)
$$

donde $\mathrm{R}$ es la matriz de requerimientos de empleo, que se define como:

$$
\mathrm{R}=\mathrm{K} * \mathrm{Z}
$$

siendo $\mathrm{Z}$ la matriz diagonal resultante de transformar el vector de demanda final regional, expresado en pesetas constantes de $1975^{3}$. K puede notarse como:

$$
\mathrm{K}=\mathrm{N} *\left(\mathrm{I}-\mathrm{A}_{\mathrm{r}}\right)^{-\mathrm{J}}
$$

donde $A_{r}$ es la matriz de coeficientes tecnicos regionales ${ }^{4}$ y $\mathrm{N}$ es la matriz diagonal resultante de transformar el vector $\mathrm{n}$ de coeficientes directos de empleo, que se obtiene a partir de:

$$
\mathrm{n}_{\mathrm{j}}=\mathrm{l}_{\mathrm{j}} / \mathrm{X}_{\mathrm{j}}
$$

donde $1_{\mathrm{j}}$ es número de trabajadores efectivamente empleados en el sector $\mathrm{j}$, y $\mathrm{X}_{\mathrm{j}}$ es su Output total regional, expresado en pesetas constantes de 1975 (véase nota 4).

Al igual que sucedia con los requerimientos de inputs intermedios, las variaciones totales en los requerimientos de empleo, medidas por (5) pueden ser debidas a tres factores: cambios en la estructura tecnológica (incorporados en $\mathrm{A}_{\mathrm{r}}$ ), cambios en la estructura de la demanda final, manteniendo constante su volumen, y cambios en la propia cuantía de la demanda (estos dos factores estan incluidos en Z).

En el presente trabajo vamos a considerar los cambios totales medidos por (5) y aquellos debidos al cambio técnico, medidos por:

$$
[\mathrm{K}(1980)-\mathrm{K}(1975)] * \mathrm{Z}(1975)^{5}
$$

3. Para deflactar hemos utilizado los deflactores e índices de precios sectoriales extraídos del Informe Anual del Banco de España de 1982.

4. No pretendemos un análisis de los hipotéticos efectos sobre el empleo de una sustitución de importantes por producción regional.

5. En el caso de los requerimientos de empleo se ha optado por mantener constante la demanda correspondiente a 1975 siguiendo el criterio utilizadø por Sáez, F. et al. (1991a) y (1991b) y Sáez F. (1992). Puede comprobarse como las diferencias en los resultados son mínimas si mantuviésemos constante la demanda de 1980 . 
Finalmente compararemos la evolución sectorial del contenido y difusión tecnológicos y de los requerimientos de empleo, al objeto de analizar el tipo de vinculación existente entre estas variables en los sectores tecnológicamente innovadores.

\section{PRECISIONES METODOLÓGICAS DEL TRABAJO}

Antes de plantear los resultados más relevantes del trabajo, conviene hacer una precisión que consideramos importante. Las Tablas Input-Output de los años 1975 y 1980 presentan un distinto grado de desagregación sectorial. De tal manera que para 1975 se consideraban 30 sectores, al ser el resultado de la unión de las dos tablas con diferente definición de los sectores realizadas para Andalucía Oriental y Occidental para este mismo año. La tabla de 1980 tiene un nivel de desagregación sectorial de 64 sectores.

Por ello, para llevar a cabo la comparación de las tablas de 1975 y 1980 hemos tenido que reducir el número de sectores de 1980 a sólo los 30 contemplados en las tablas de 1975, siguiendo los criterios especificados en las mismas (La lista de los sectores se encuentra en el APENDICE I). Somos conscientes de la pérdida de información que ello implica pero nos vemos obligados a renunciar a ella en aras de poder llevar a cabo la primera parte del presente trabajo.

Un problema semejante surge cuando abordamos el estudio del efecto sobre el empleo en la segunda parte del estudio. Las Cuentas Regionales publicadas conjuntamente con las Tablas Input-Output de ambos años sólo permiten obtener un vector de empleo desagregado a 28 sectores. Esto nos ha obligado a hacer una nueva agregación, resultando únicamente 28 sectores con la consiguiente pérdida adicional de información ${ }^{6}$ (APENDICE II).

La utilizacion de los coeficientes técnicos totales para el análisis de la difusión del cambio tecnológico se justifica porque la difusión, no es sólo de la tecnología generada en la región, sino también de aquella incorporada en inputs procedentes de otras áreas. Al analizar los efectos sobre el empleo, han de utilizarse sólo los coeficientes técnicos regionales, porque lo que se pretende es estudiar el efecto del cambio tecnológico en el empleo regional.

6. Aunque se podrían haber obtenido los vectores de empleo a partir de otras fuentes que permitiesen mantener los ya reducidos 30 sectores, optamos por usar los aportados por la Contabilidad Regional para reducir los problemas de definición de sectores. 


\section{ANÁLISIS DE LOS RESULTADOS.}

\subsection{Análisis de Ia difusión del cambio tecnológico.}

El cambio y el progreso técnico deben implicar un ahorro en los costes de produccion medidos en los requerimientos de insumos primarios. Los bienes intermedios del proceso productivo también pueden modificarse como consecuencia del avance tecnológico. Por ello es necesario estudiar las variaciones experimentadas por los requerimientos de inputs intermedios y determinar así las líneas generales del cambio en la estructura del sistema productivo regional (Fanjul et al. (1975) pág. 43).

A la hora de analizar los resultados obtenidos hay que tener presente de forma clara que el período que comprendemos en nuestro estudio es una época caracterizada por una grave crisis económica generalizada a toda España y especialmente a Andalucía. Pero, quizás lo más significativo, es un importante reajuste en ramas concretas de la actividad industrial (IFA (1991) pág. 83). Este es el panorama económico general en el que hay que inscribir el análisis de los datos que a continuación se especifican.

\subsubsection{Análisis General}

En la Tabla 1 aparecen los porcentajes que respecto a la producción total bruta $\left(\mathrm{PBT}_{\mathrm{t}}\right)$ suponen los bienes de uso intermedio en la demanda final. Estos porcentajes se calculan respecto a un producto bruto total definido como:

$$
\operatorname{PBT}_{\mathrm{t}}=\mathrm{q}_{\mathrm{t}}^{*}+\mathrm{z}_{80}(\mathrm{t}=1975,1980)
$$

en el que $\mathrm{q}_{\mathrm{t}}{ }^{*}$ representa el total de los requerimientos de bienes intermedios y $\mathrm{z}_{80}$ la demanda final de 1980.

A la luz de los datos puede concluirse que el peso relativo de los requerimientos intermedios ha crecido 0,71 puntos en el período considerado. Un mayor volumen de requerimientos intermedios puede deberse a dos causas fundamentalmente. O bien una mayor importancia del reempleo $\left(a_{j j}{ }^{80}>a_{j j}{ }^{75}\right)$, o una mayor dependencia sectorial $\left(a_{j i}{ }^{80}>a_{j i}{ }^{75}\right)$. El caso de un mayor reempleo puede identificarse como un aumento del grado de especialización dentro del propio sector, mientras que una mayor dependencia sectorial puede reflejar tambien un cambio 
en la propia estructura productiva que puede llegar a alterar la composición de los inputs intermedios utilizados.

\section{TABLA 1}

Producción bruta, demanda final y requerimientos de bienes intermedios necesarios para satisfacer la demanda final de 1980,con las tecnologías de los años 1975 y 1980. (en miles de millones de ptas.)

\begin{tabular}{lrrrrr}
\hline & 1975 & $(\%)$ & & 1980 & $(\%)$ \\
\cline { 2 - 3 } \cline { 5 - 6 } Demanda Final & $2.309,18$ & 61,52 & & $2.309,18$ & 60,81 \\
Requerimientos Intermedios & $1.444,20$ & 38,48 & & $1.488,35$ & 39,19 \\
Producción Total Bruta $\left(\mathrm{z}_{80}\right)$ & $3.753,38$ & 100,00 & & $3.797,53$ & 100,00 \\
\hline
\end{tabular}

A la hora de seleccionar los sectores más interesantes para estudiar el efecto del cambio tecnológico en Andalucía hemos tratado de aunar los criterios de selección recogidos en Buesa, M y Molero J. (1992) y Palma, L. Martín, J. L. y Villar, C. (1992) (Ver ANEXO III). En las Tablas 2, 3, 4, y 5 se recogen datos relativos a los 12 sectores finalmente seleccionados.

Los casos de aumento en reempleo se han producido en los sectores de Química Básica, Materiales de Construcción, Metálicas Básicas, Agua, Gas y Electricidad, Transporte y Comunicaciones, y Banca y Seguros.

\subsubsection{Sectores específicos.}

La Tabla 2 recoge las tasas de variación de outputs intermedios suministrados por cada sector seleccionado. Los sectores más expansivos han sido Maquinaria y Equipos de Transporte y Transformados Metálicos de manera destacada. Con un crecimiento más moderado están los sectores de Agua, Gas y Electricidad, Minas y Canteras, Transportes y Comunicaciones, Química Básica y Otras Químicas. De los sectores seleccionados es solamente Otras Manufactureras el que muestra una tendencia a la reducción.

En la Tabla aparecen claros los efectos de un aumento en la especialización del sector químico debido a la sustitución de materiales tradicionales por derivados plásticos en algunas actividades claves como la construcción, fabricación de maquinarias o textiles, así como el mayor uso de los abonos en la agricultura andaluza. Asímismo es de reseñar el crecimiento de las industrias energéticas que experimenta un aumento del output intermedio debido a un proceso de sustitución de fuentes energéticas. Del mismo modo puede interpretarse el sector de 
transformados metálicos y de maquinaria de todo tipo que refleja la fuerte especialización intrasectorial.

La práctica totalidad de los sectores analizados presenta una tasa de variación porcentual tanto en lo referente a inputs como a outputs intermedios superior a la media, que puede estimarse en un $3,05 \%$.

Se ha constatado que todos los sectores presentados en la Tabla 3 cuyos inputs intermedios aumentan experimentan una disminución de las necesidades de inputs no regionales, salvo el sector Química Básica en el que se puede apreciar un notable aumento de los requerimientos de inputs no andaluces. Este fenómeno indica que el aumento del output de dicho sector ha sido posible básicamente a través de importaciones de bienes no andaluces.

TABLA 2

Sectores productivos más significativos ordenados según su tasa porcentual de variación (1980-1975) de los outputs intermedios

a) Sectores cuyos outputs intermedios aumentan

S20. Maq. y Equipos de Transporte

Tasa (\%)

S19. Transformados Metálicos

291,11

S22. Agua, Gas y Electricidad

210,37

S03. Minas y Canteras

52,66

S26. Transporte y Comunicaciones

41,19

S15. Quimica Básica

36,29

S16. Otras Químicas

25,98

S17. Materiales de Construcción

23,77

S27. Banca y Seguros

16,44

S23. Construcción y Obras Públicas

9,78

S18. Metálicas Básicas

5,13

2,53

b) Sectores cuyos outputs intermedios disminuyen

S21. Otras Manufactureras

$-40,42$ 
TABLA 3

Sectores productivos más significativos ordenados según su tasa porcentual de variación (1980-1975) de los inputs intermedios

a) Sectores cuyos inputs intermedios aumentan

S27. Banca y Seguros

S19. Transformados Metálicos

S22. Agua, Gas y Electricidad

S26. Transporte y Comunicaciones

S21. Otras Manufactureras

S17. Materiales de Construcción

S15. Química Básica

S03. Minas y Canteras

S23. Construcción y Obras Públicas

b) Sectores cuyos inputs intermedios disminuyen

S16. Otras Químicas

S20. Maq. y Equipos de Transporte

S18. Metálicas Básicas
Tasa $(\%)$

317,16

217,63

135,43

119,02

87,02

62,26

56,23

45,59

3,31

$-18,52$

$-9,78$

$-3,80$

De entre los sectores que experimentan una disminución en los requerimiento de inputs intermedios, Maquinaria y Equipos de Transporte y Metálicas Básicas pertenecen a la Industria pesada, cuyo output estaba en claro retroceso en e periodo analizado.

En las Tablas 4 y 5 puede observarse el peso relativo de los outputs । inputs sobre los totales en los respectivos sectores. Se observa una tendenci: al aumento en la participacion de los sectores Minas y Canteras, Químic. Básica, Transformados Metálicos, Agua, Gas y Electricidad, Maquinaria : Equipo de Transporte y Transportes y Comunicaciones. Por el contrario, lo sectores relacionados con Construcción y Obras Públicas mantienen aproxima damente la misma participación. 
TABLA 4

Porcentaje de output intermedio de cada sector sobre el total

Sectores

\begin{tabular}{|c|c|}
\hline 1975 & 1980 \\
\hline 10,94 & 14,76 \\
\hline 8,66 & 10,42 \\
\hline 2,41 & 2,85 \\
\hline 3,38 & 3,76 \\
\hline 4,63 & 4,53 \\
\hline 1,16 & 3,44 \\
\hline 1,31 & 4,91 \\
\hline 0,32 & 0,18 \\
\hline 2,84 & 4,15 \\
\hline 1,01 & 1,01 \\
\hline 5,70 & 7,42 \\
\hline 5,18 & 5,43 \\
\hline
\end{tabular}

S3. Minas y Canteras

S15. Química Básica $8,66 \quad 10,42$

S16. Otras Químicas

S17. Materiales de Construcción 3,38

S18. Metálicas Básicas

S19. Transformados Metálicos

S20. Maq. y Equipos de Transporte

S21. Otras Manufactureras

S22. Agua, Gas y Electricidad

S23. Construcción y Obras Públicas

S26. Transporte y Comunicaciones

S27. Banca y Seguros

5,18

\section{TABLA 5}

Porcentaje de input intermedio de cada sector sobre el total

Sectores

S3. Minas y Canteras

S15. Química Básica

S16. Otras Químicas

S17. Materiales de Construcción

S18. Metálicas Básicas

S19. Transformados Metálicos

S20. Maq. y Equipos de Transporte

S21. Otras Manufactureras

S22. Agua, Gas y Electricidad

S23. Construcción y Obras Públicas

S26. Transporte y Comunicaciones

S27. Banca y Seguros

\begin{tabular}{|c|c|}
\hline 1975 & 1980 \\
\hline 0,16 & 0,23 \\
\hline 4,85 & 7,36 \\
\hline 0,89 & 0,70 \\
\hline 1,35 & 2,12 \\
\hline 3,41 & 3,18 \\
\hline 0,06 & 0,17 \\
\hline 7,39 & 6,47 \\
\hline 0,15 & 0,27 \\
\hline 1,15 & 2,63 \\
\hline 10,60 & 10,62 \\
\hline 1,94 & 4,12 \\
\hline 0,30 & 123 \\
\hline
\end{tabular}


En lo referente a la evolucion de los inputs intermedios, crece la participación de Química Básica, Agua, Gas y Electricidad, Transporte y Comunicaciones y Banca y Seguros, permaneciendo muy estables los sectores relacionados con la Construcción y Obras Públicas.

\subsection{Análisis de los niveles de empleo en los sectores potencialmente innovadores}

La Tabla 6 recoge los índices sectoriales de contenido tecnológico regional y las variaciones de empleo, para el periodo de estudio considerado, de los 28 sectores económicos. Estos datos permiten una primera aproximación al fenómeno del impacto del cambio tecnológico en el nivel de empleo.

Suponemos que el mayor grado de contenido tecnólogico indica una mayor utilización de procesos productivos tecnológicamente avanzados, tanto directa como indirectamente. La utilización, por parte de un determinado sector productivo, de inputs derivados de procesos que requieren un alto nivel tecnológico, implica la utilización indirecta de la tecnología que dichos inputs incorporan. Así, es posible que sectores cuyo proceso productivo no sea tecnológicamente avanzado, en cambio sus inputs intermedios lleven incorporado un alto componente técnico. El contenido tecnológico constituye por tanto una primera aproximación a la medicion deI nivel de tecnología incorporado en cada sector.

Al ordenar los sectores de mayor a menor contenido tecnológico en 1980, se observa cómo entre los 14 sectores con un índice superior a $75 \%$ se encuentran los doce seleccionados como potencialmente innovadores. La práctica totalidad de estos sectores han experimentado una diferencia positiva en los índices de contenido tecnológico, constituyendo excepciones Metálicas Básicas, Transformados Metálicos, Construcción y Obras Públicas y Otros Servicios Personales e Industriales.

Con un índice inferior a 20 tenemos los sectores de Aceite y Grasas Vegetales, Molinería Panadería y Piensos y Sacrificio de Ganado e Industrias Cárnicas, que no obstante presentan una diferencia positiva.

Podemos extraer como primera conclusión que los sectores potencialmente innovadores han incorporado de hecho, una mayor carga tecnológica en sus procesos productivos, ya sea por la vía directa o por la indirecta. Puede indicarse tambien, que de entre los 14 sectores destacados sólo tres tenían un índice inferior a 75\% en 1975, a saber: S19 (Otras manufactureras), S20 (Agua, gas y electricidad) y S28 (Administración Pública y Defensa). De ellos, los dos 
primeros están en el grupo de sectores potencialmente innovadores. Así pues a excepción de estos dos, los sectores potencialmente innovadores han estado presentes entre los sectores con un mayor aporte tecnológico durante el período considerado.

Otra importante conclusión que se deriva de los datos presentados en la tabla 6 es la profunda estabilidad en el grupo en el que se incluye cada sector. También se observa una especie de "dualidad" en los sectores estudiados. Tomando como referencia el valor crítico del $75 \%$, los 14 sectores que lo superan están en unos porcentajes muy cercanos (solo 19 puntos de diferencia entre el primero y el decimocuarto). Por contra los otros 14 sectores muestran una gran dispersión (aproximadamente 44 puntos de diferencia) además de haber un importante gap entre el decimocuarto $(78,73 \%)$ y el decimoquinto $(55,08 \%)$.

En la Tabla 7 se analizan las diferentes causas de variacion de los requerimientos de empleo totales. La primera columna recoge el cambio de empleo total derivado de la matriz resultante de la ecuación (5), por columnas. A continuación se recogen las diferencias ocasionadas por cada uno de los tres factores anteriormente considerados. Podemos así conocer cuáles son los sectores responsables del cambio tecnológico, aquellos que cambian sus requerimientos de empleo por unidad de demanda final (millón de pesetas), aunque no sean ellos mismos los que se vean afectados por dichos cambios. Se trata en definitiva de evaluar el efecto del cambio técnico en un sector sobre el empleo en todos los sectores de la economía, incluido el mismo (Sáez, F. et al. 1991 pág 219).

El empleo potencial de la economía andaluza en 1975 ascendía a 1.785 .594 personas, de tal modo que el descenso motivado por el cambio técnico (- 303.287) supone una tasa media anual del $-3.4 \%$ en el período 1975-80. Si la estructura y el volumen de la demanda final de 1975 se hubieran mantenido constante, éste hubiera sido el descenso del empleo potencial.

De los 28 sectores considerados, sólo cinco presentan tasas de variación de empleo positivas debidas al cambio técnico o sea que el avance tecnológico en estos sectores ha aumentado el empleo en la economía. Se trata de S2, S7, S17, S25 y S27. Este último, "Otros Servicios Personales e Industriales", es el más importante (16.621). El efecto de este sector es básicamente sobre si mismo y es prácticamente despreciable el impacto sobre los otros. Algo parecido podría decirse del S17, "Transformados Metálicos", así como del S25, "Banca y Seguros", cuyo efecto positivo recae básicamente sobre sí mismo y en una parte significativa sobre S27. 


\section{Tabla 6}

Indices Sectoriales de Contenido Tecnológico y Variación del Empleo (1975-1980)

\begin{tabular}{|c|c|c|c|c|}
\hline & 1975 & 1980 & Diferencia & Var. empl. (\%) \\
\hline $\mathrm{S} 25$ & 96,01 & 97,94 & 1,93 & 20,73 \\
\hline S16 & 98,96 & 95,59 & $-3,37$ & $-46,23$ \\
\hline S18 & 78,79 & 93,76 & 14,97 & 18,77 \\
\hline S14 & 90,62 & 93,71 & 3,09 & $-33,54$ \\
\hline S3 & 88,35 & 92,37 & 4,02 & 15,04 \\
\hline S20 & 72,62 & 87,26 & 14,64 & 57,19 \\
\hline S17 & 88,33 & 87,17 & $-1,16$ & $-16,96$ \\
\hline S15 & 78,13 & 86,53 & 8,40 & 121,61 \\
\hline $\mathrm{S} 21$ & 88,79 & 85,23 & $-3,56$ & $-19,00$ \\
\hline $\mathrm{S} 22$ & 76,85 & 84,83 & 7,98 & 31,13 \\
\hline S27 & 81,58 & 80,57 & $-1,01$ & 29,89 \\
\hline S24 & 78,80 & 79,01 & 0,21 & $-16,05$ \\
\hline S19 & 67,98 & 78,88 & 10,90 & $-31,58$ \\
\hline $\mathrm{S} 28$ & 57,55 & 78,73 & 21,18 & $-9,96$ \\
\hline S26 & 59,73 & 55,08 & $-4,65$ & 40,84 \\
\hline $\mathrm{S} 2$ & 73,33 & 52,59 & $-20,74$ & $-2,01$ \\
\hline $\mathrm{S} 10$ & 28,79 & 39,34 & 10,55 & $-45,28$ \\
\hline S1 & 55,43 & 39,21 & $-16,22$ & $-13,58$ \\
\hline S13 & 35,84 & 38,75 & 2,91 & 4,59 \\
\hline $\mathrm{S} 12$ & 36,72 & 35,18 & $-1,54$ & - 17,90 \\
\hline S9 & 13,40 & 33,90 & 20,50 & $-38,80$ \\
\hline $\mathrm{S} 23$ & 32,00 & 32,39 & 0,39 & $-8,32$ \\
\hline Sll & 29,90 & 27,51 & $-2,39$ & 0,18 \\
\hline S6 & 28,83 & 26,52 & $-2,31$ & $-19,93$ \\
\hline S5 & 11,27 & 24,60 & 13,33 & 122,77 \\
\hline S7 & 6,31 & 18,27 & 11,96 & $-22,98$ \\
\hline S8 & 15,78 & 16,10 & 0,32 & $-4,32$ \\
\hline S4 & 4,39 & 10,95 & 6,56 & 2,42 \\
\hline
\end{tabular}

Fuente: TIOA 1975, 1980 y elaboración propia. 


\section{Tabla 7}

Variaciones Totales en los Requerimientos de Empleo y sus Factores Responsables

\begin{tabular}{|c|c|c|c|c|}
\hline & Totales & Tecnología & Estructura D.F. & Volumen D.F. \\
\hline Sl & $-36.675,0$ & $-40.362,0$ & $-39.657,9$ & $43.345,0$ \\
\hline $\mathrm{S} 2$ & $-535,9$ & $6.287,2$ & $-11.676,6$ & $4.843,5$ \\
\hline S3 & 613,8 & $-1.403,6$ & $1.145,1$ & 872,3 \\
\hline S4 & 799,5 & $-3.737,1$ & $-1.737,2$ & $6.273,8$ \\
\hline S5 & $11.195,7$ & $-431,0$ & $7.850,8$ & $3.776,0$ \\
\hline S6 & $-8.257,0$ & $-12.505,8$ & $-1.916,0$ & $6.164,9$ \\
\hline S7 & $-23.360,9$ & $2.995,5$ & $-40.903,1$ & $14.546,7$ \\
\hline S8 & $-2.545,1$ & $-14.995,5$ & $1.973,3$ & $10.477,2$ \\
\hline S9 & $-10.533,8$ & $-10.627,0$ & $-2.994,3$ & $3.087,5$ \\
\hline $\mathrm{S} 10$ & $-43.341,3$ & $-56.883,1$ & $3.808,2$ & $9.733,5$ \\
\hline S11 & 85,8 & $-9.240,8$ & 566,5 & $8.760,0$ \\
\hline $\mathrm{S} 12$ & $-3.789,2$ & $-2.625,2$ & $-4.393,0$ & $3.229,0$ \\
\hline $\mathrm{S} 13$ & 488,3 & $-553,1$ & $-1.023,1$ & $2.064,5$ \\
\hline S14 & $-9.349,4$ & $-18.975,5$ & $6.183,6$ & $3.442,6$ \\
\hline S15 & $10.415,2$ & $-3.382,7$ & 270,2 & $3.527,7$ \\
\hline $\mathrm{S} 16$ & $-6.846,6$ & $-7.079,6$ & $-1.246,9$ & $1.479,9$ \\
\hline $\mathrm{S} 17$ & $-3.611,8$ & $7.957,3$ & $-14.855,8$ & $3.286,8$ \\
\hline $\mathrm{S} 18$ & $9.285,6$ & $-3.672,7$ & $2.040,2$ & $10.918,0$ \\
\hline S19 & $-1.525,4$ & $-2.166,7$ & 27,2 & 614,1 \\
\hline $\mathrm{S} 20$ & $5.266,5$ & $-3.505,4$ & $1.023,9$ & $7.747,9$ \\
\hline S21 & $-40.659,1$ & $-45.220,8$ & $-27.638,3$ & $32.200,0$ \\
\hline $\mathrm{S} 22$ & $48.793,3$ & $-22.895,0$ & $31.655,2$ & $40.033,1$ \\
\hline $\mathrm{S} 23$ & $-10287,7$ & $-43.783,4$ & $12.427,6$ & $21.068,1$ \\
\hline S24 & $-11.422,3$ & $-27.948,8$ & $5.422,0$ & $11.104,4$ \\
\hline $\mathrm{S} 25$ & $1.549,5$ & $6.228,1$ & $-6.355,8$ & $1.677,1$ \\
\hline$\$ 26$ & $40.628,2$ & $-170,7$ & $14.756,6$ & $26.042,3$ \\
\hline S27 & $37.097,4$ & $16.621,1$ & $-9.483,6$ & $29.960,0$ \\
\hline $\mathrm{S} 28$ & $-10.648,8$ & $11.220,6$ & $-17.306,5$ & $17.878,4$ \\
\hline TOT & $-57.171,1$ & $-303.287,0$ & $-82.037,9$ & $328.154,3$ \\
\hline
\end{tabular}

Fuente: TIOA 1975, 1980 y elaboración propia. 
Quedan por tanto 23 sectores en los que el cambio tecnológico tiene un: incidencia negativa sobre el empleo potencial. Diez sectores S1, S8, S9, S10 S14, S21, S24 y S28 destacan con reducciones en valor absoluto por encima d6 10.000 empleos. Con un efecto negativo muy acusado destacan los sectore: "Agropecuario y Forestal" (40.362), "Otras Alimentarias" (56.883), "Construccione: y Obras Públicas" (45.221) y "Transportes y Comunicaciones" (27.948).

Podemos detenernos en examinar si los efectos negativos sobre el emplec recaen sobre el propio sector o se distribuyen entre otros sectores. "Agropecuaric y Forestal" ejerce una fuerte incidencia sobre él mismo y sobre "Comercio y Otros Servicios Personales e Industriales. "Otras Alimentarias afecta muy negativamente al "Sector Agropecuario y Forestal" y a "Otros Servicios Persona. les e Industriales". "Construcciones y Obras Públicas" incide fuertemente sobre sí mismo y sobre "Materiales de Construcción", y Otros Servicios Personales $\epsilon$ Industriales". "Transportes y Comunicaciones" afecta sobre todo al propio sector y a "Otros Servicios Personales e Industriales".

Por otro lado, como se observa en la Tabla 6 los 14 sectores con mayor índice de contenido tecnológico (índice mayor a 75) presentan una evolución más favorable en sus requerimientos de empleo que el resto de los sectores.

En lo que respecta a los sectores potencialmente innovadores (todos ellos deutro del grupo de mayor contenido tecnológico), pueden señalarse comc excepciones los comportamientos de S16, S17 y S21, los cuales disminuyen sus requerimientos de empleo y también su nivel de contenido tecnológico. Los sectores S14, S24 y S19 muestran un comportamiento especialmente interesante pues sus requerimientos de empleo disminuyen pese a aumentar su contenido tecnológico.

\section{CONCLUSIONES.}

Se ha pretendido con el presente trabajo una aproximación general al conocimiento del grado de difusión tecnológica en una serie de sectores de la economía andaluza que según los criterios utilizados presentan un especial componente tecnológico. Somos conscientes de que un estudio mas pormenorizado, que atendiera al análisis en profundidad de subsectores, arrojarla un mejor conocimiento de la realidad económica regional en el período considerado, en cuanto a su vinculación con el fenómeno del cambio tecnológico. 
No obstante se observa, si nos circunscribimos a los sectores seleccionados, un comportamiento similar al experimentado por el conjunto de los sectores económicos nacionales en la época que va de 1962 a 1970 (Fanjul et al. 1975). Si consideramos que esta época corresponde a un período de expansión económica, podría concluirse que los sectores andaluces analizados, potencialmente innovadores, han superado la etapa 1975-1980, a todas luces recesiva, en unas condiciones favorables.

Como conclusión del apartado 4.2 puede destacarse la existencia observada de una cierta correlación entre la evolucion del contenido tecnológico y de las variaciones de empleo en la mayoría de los sectores considerados. Los datos existentes no nos permiten comprobar si la correlación mejoraría al desfasar un quinquenio los valores del empleo ni tampoco analizar un período mas amplio. Esto nos permitiría afinar las conclusiones pues parece lógico pensar que existe un período de maduración de las inversiones que reporta una mejora en la competitividad de las empresas tecnológicamente avanzadas y por tanto en los niveles de empleo requeridos, como indica Sáez (Sáez, F. 1992).

La aparición de una nueva Tabla Input-Output andaluza que permita extender el período de estudio así como una profundización e interrelación en diversos aspectos tocados aquí con un carácter aproximativo, propiciará un mejor conocimiento de la realidad analizada en este trabajo. 


\section{APÉNDICE 1}

\section{TABLA DE 30 SECTORES CONTENIDOS EN LAS TABLAS DE 1975, A LOS QUE SE AGREGARON LOS 64 DE 1980.}

S1 Agropecuario y forestal

S2 Pesca

S3 Minas y canteras

S4 Sacrificio de ganado e industrias cárnicas

S5 Industrias lácteas

S6 Industrias conserveras

S7 Aceites y grasas vegetales

S8 Molinería, panadería y piensos

S9 Industrias del azúcar

S10 Otras alimentarias

S11 Alcoholes, vinos y tabaco

S12 Industria textil y del calzado

S13 Industria de la madera y corcho

S14 Transformados y fabricación de papel y cartón, artes gráficas y editoriales

S15 Industrias químicas básicas

S16 Otras industrias químicas.

S17 Industrias de materiales para la construcción

S18 Metálicas básicas

S19 Transformados metálicos

S20 Maquinaria y equipos de transporte

S21 Otras manufactureras

S22 Agua, gas y electricidad

S23 Construcciones y obras públicas

S24 Comercio

S25 Hostelería y restauración

S26 Transportes y comunicaciones

S27 Banca y seguros

S28 Enseñanza y sanidad

S29 Otros servicios personales e industriales

S30 Administración pública y defensa 


\section{APÉNDICE 2}

TABLA DE LOS 28 SECTORES A LOS QUEDAN AGREGADAS FINALMENTE LAS TABLAS AL INTRODUCIR EL VECTOR DE EMPLEO

S1 Agropecuario y forestal

S2 Pesca

S3 Minas y canteras

S4 Sacrificio de ganado e industrias cárnicas

S5 Industrias lácteas

S6 Industrias conserveras

S7 Aceites y grasas vegetales

S8 Molinería, panadería y piensos

S9 Industrias del azúcar

S10 Otras alimentarias, alcoholes, vinos y tabaco

S11 Industria textil y del calzado

S12 Industria de la madera y corcho

S13 Transformados y fabricación de papeI y cartón, artes gráficas y editoriales

S14 Industrias químicas

S15 Industrias de materiales para la construcción

S16 Metálicas básicas

S17 Transformados metálicos

S18 Maquinaria y equipos de transporte

S19 Otras manufactureras

S20 Agua, gas y electricidad

S21 Construcciones y obras públicas

S22 Comercio

S23 Hostelería y restauración

S24 Transportes y comunicaciones

S25 Banca y seguros

S26 Enseñanza y sanidad

S27 Otros servicios personales e industriales

S28 Administración pública y defensa 


\section{APÉNDICE 3}

\section{TABLA DE LOS 12 SECTORES POTENCIALMENTE INNOVADORES}

S3. Minas y Canteras

S15. Química Básica

S16. Otras Químicas

S17. Materiales de Construcción

S18. Metálicas Básicas

S19. Transformados Metálicos

S20. Maq. y Equipos de Transporte

S21. Otras Manufactureras

S22. Agua, Gas y Electricidad

S23. Construcción y Obras Públicas

S26. Transporte y Comunicaciones

S27. Banca y Seguros

\section{BIBLIOGRAFÍA.}

AFRASIABI, A. Y CASLER (1991): Product-mix and Technological change within the Leontief Inverse Journal of Regional Science, Vol. 31, n· 2. pág. 147-160.

AA.VV. (1982): Tablas Input-output y cuentas regionales. Teorías, métodos y aplicaciones. Instituto de Desarrollo Regional. Universidad de Sevilla. Sevilla.

BANCO DE ESPAÑA (1982): Informe Anual. Madrid.

BUESA, M. Y MOLERO, J. (1992) Patrones de Cambio Tecnológico y Política Industrial. Un estudio de las empresas innovadoras madrileñas. Biblioteca Civitas Economía y Empresa. Madrid.

Cuentas Económicas de Andalucía. Tabla input-output y contabilidad regional. (2 Vol.). Banco de

Bilbao-Andalucía; Junta de Andalucía; Cajas rurales de Andalucía; Instituto de Desarrollo Regional de la Universidad de Sevilla. 1985.

FANJUL, O. et al. (1975): Cambios en la estructura interindustrial de la economía española. 1962-1970: Una primera aproximación. Fundación del Instituto Nacional de Industria. Madrid. INSTITUTO DE FOMENTO DE ANDALUCÍA (1991): El Sector Industrial en Andalucía. Consejería de Economía y Hacienda. Sevilla.

McNICOLL, I. H., y BAIRD, R. G. (1980): Empirical Applications of regional Input-Output Analysis: A Case Study of Shetland. Journal of the Operational Research Society. Vol 36, pág. 983-991. 
PALMA MARTOS, L. MARTÍN NAVARRO, J. L. y VILLAR RODRÍGUEZ, C. (1992): La Política Tecnológica en Andalucía: Una década de actuaciones". Comunicación presentada a la VI Reunión Anual ASEPELT-ESPAÑA. Granada.

PULIDO, A. y FONTELA, E. (1993): Análisis input-output: Modelos, datos y aplicaciones. Ed. pirámide.

RAMÍREZ SOBRINO, JESÚS N. (1993): El Análisis cuantitativo de la economía regional: los modelos econométricos regionales. Publicaciones E.T.E.A., Córdoba.

SÁEZ, F. et al. (1991a): Tecnología y Empleo en España: Situación y perspectivas. Secretaria de Estado de Economía y Hacienda. Madrid.

SÁEZ, F. et al. (1991b): Tecnología, empleo y estructura productiva. Economía Industrial. n· 277. pág 205-216.

SÁEZ, F. (1992): Cambio Técnico y dinámica de empleo. En Ruesga, S. (1992) Economía y Trabajo. Pirámide. Madrid.

SATO, R. y RAMACHANDRAN, R. (1980): Measuring the impact of Technical Progress on the Demand for Intermediate Goods: A Survey. Journal of Economic Literature, Vol. XVIII, pág. 1003-1024.

SEGURA, J. y RESTOY, F. (1986): Una explotación de las tablas input-output de la economía española para 1975 y 1980 Documento de trabajo de la Fundación Empresa Pública, ก. 8608.81 pág.

STONEMAN, P. (1983): The Economics Analysis of Technological Change. Oxford University Press. Oxford.

TABLA INPUT-OUTPUT Y CONTABILIDAD REGIONAL DE LA ECONOMÍA ANDALUZA. Departamento de Política Económica; Instituto de Desarrollo Regional, Universidad de Sevilla; Banco de Bilbao. 1979. 